\title{
PIK3CA mutation correlates with mTOR pathway expression but not clinical and pathological features in Fibro-adipose vascular anomaly (FAVA)
}

Yumiko Hori ${ }^{1,2}$, Katsutoshi Hirose ${ }^{3}$, Michio Ozeki $^{4}$, Kenji Hata $^{5}$, Daisuke Motooka ${ }^{6}$, Shinichiro Tahara', Takahiro Matsui ${ }^{1}$, Masaharu Kohara ${ }^{1}$, Hiroki Higashihara ${ }^{7}$, Yusuke Ono ${ }^{7}$, Kaishu Tanaka ${ }^{7}$, Satoru Toyosawa ${ }^{3}$ and Eiichi Morii ${ }^{1 *}$

\begin{abstract}
Background: Fibro-adipose vascular anomaly (FAVA) is a rare and new entity of vascular anomaly. Activating mutations in the phosphatidylinositol-4,5-bisphosphate 3-kinase catalytic subunit alpha (PIK3CA) gene were identified at a frequency of $62.5 \%$ in FAVA cases. The PIK3CA mutations excessively activate mammalian target of rapamycin (mTOR) pathway, which promotes angiogenesis and lymphangiogenesis, implying that PIK3CA mutations may act as drivers of FAVAs. This study investigated the correlations between PIK3CA mutational status, clinicopathological features and immunohistochemical expression of the mTOR pathway in a series of FAVA.

Methods: We retrospectively evaluated the clinical and pathological findings of four FAVA cases. We performed next-generation sequencing (NGS) with a custom panel of genes associated with the mTOR pathway and genes responsible for other vascular anomalies; followed by direct sequencing and immunohistochemical analysis of the mTOR pathway.

Results: Two PIK3CA-mutation cases and two PIK3CA-wild-type (wt) cases exhibited similar typical clinical features of FAVA. Histological analysis revealed venous malformation, lymphatic malformation, nerves containing enlarged abnormal vessels and fibrofatty tissue were observed regardless of PIK3CA mutational status. In contrast to clinical and histological findings, the immunohistochemical expression of activated AKT and mTOR that are upstream of the mTOR pathway was detected in abnormal vessels of PIK3CA-mutation cases but not in those of PIK3CA-wt cases. However, activated eukaryotic translation initiation factor 4E-binding protein 1 (4EBP1) and ribosomal protein S6 kinase 1 (S6K1), both of which are downstream effectors of the mTOR pathway, were expressed in abnormal vessels of both PIK3CA-mutation and PIK3CA-wt cases. Furthermore, targeting NGS did not find any common genetic mutations involved in the mTOR pathway among PIK3CA-wt cases.
\end{abstract}

\footnotetext{
*Correspondence: morii@molpath.med.osaka-u.ac.jp

1 Department of Pathology, Osaka University Graduate School of Medicine,

2-2 Yamadaoka, 565-0871 Suita, Osaka, Japan

Full list of author information is available at the end of the article
}

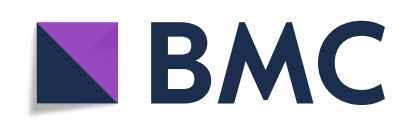

(c) The Author(s). 2022, corrected publication 2022. Open Access This article is licensed under a Creative Commons Attribution 4.0 International License, which permits use, sharing, adaptation, distribution and reproduction in any medium or format, as long as you give appropriate credit to the original author(s) and the source, provide a link to the Creative Commons licence, and indicate if changes were made. The images or other third party material in this article are included in the article's Creative Commons licence, unless indicated otherwise in a credit line to the material. If material is not included in the article's Creative Commons licence and your intended use is not permitted by statutory regulation or exceeds the permitted use, you will need to obtain permission directly from the copyright holder. To view a copy of this licence, visit http://creativecommons.org/ licenses/by/4.0/. The Creative Commons Public Domain Dedication waiver (http://creativecommons.org/publicdomain/zero/1. 0/) applies to the data made available in this article, unless otherwise stated in a credit line to the data. 
Conclusions: There was no significant association between the presence of PIK3CA mutations and the clinicopathological features of FAVA, suggesting that the PIK3CA gene is not necessarily involved in the onset of FAVA. FAVAs lacking PIK3CA mutations may be caused by other gene mutations that activate 4EBP1 and S6K1.

Keywords: Fibro-adipose vascular anomaly, FAVA, PIK3CA, mTOR, Vascular anomaly, Lymphatic malformation, Venous malformation, Sirolimus

\section{Background}

Fibro-adipose vascular anomaly (FAVA) is a newly described vascular anomaly [1]. FAVA is extremely rare and occurs most commonly in the muscles of the lower extremities of young patients $[1,2]$. Histologically, FAVA is composed of venous malformation (VM), lymphatic malformation (LM), and the presence of fibro-adipose tissues with the atrophic skeletal muscle [1,3]. A recent study identified somatic and mosaic gain-of-function mutations of the phosphatidylinositol-4,5-bisphosphate 3-kinase catalytic subunit alpha (PIK3CA) gene in a subset of FAVAs (62.5\%) [4]. The identified PIK3CA mutations are p.E542K, p.E545K and p.Q546K in the helical domain (encoded within exon 9), and p.H1047R in the kinase domain (encoded within exon 20) [4]. These PIK3CA mutations are termed hotspot mutations, and are present in a subset of VMs and the majority of LMs [4-10]. PIK3CA mutations excessively activate the phosphoinositide 3-kinase (PI3K)/AKT/mammalian target of rapamycin (mTOR) pathway in the endothelial cells during vascular developments [4-10]. Activation of PI3K results in the phosphorylation of AKT (p-AKT), and pAKT phosphorylates mTOR. Furthermore, the phosphorylated form of mTOR (p-mTOR) phosphorylates downstream effectors such as eukaryotic translation initiation factor 4E-binding protein 1 (4EBP1) and ribosomal protein $\mathrm{S} 6$ kinase 1 (S6K1), ultimately promoting angiogenesis and lymphangiogenesis [11, 12]. These results highly suggested that PIK3CA mutations may act as drivers of FAVAs through activation of the PI3K/ AKT/mTOR pathway. Furthermore, the presence of PIK3CA mutations in VMs and the genotype of PIK3CA mutation in LMs correlate with both clinical severity and histological features $[8,10,13]$. However, little is known regarding the correlations among PIK3CA mutational status, the mTOR pathway activation status and clinicopathological features in FAVA. Here, we report the results of clinical, histological, immunohistochemical, and genetic analyses examining a small series of isolated FAVA cases.

\section{Methods}

Four FAVA cases with formalin-fixed paraffin-embedded (FFPE) tissues were retrieved from the pathology files of Osaka University Hospital from 2010 to 2020. A final diagnosis of FAVA was determined by consensus agreement after consideration of clinical, radiologic, and histological findings [1-3]. This study was approved by the Ethical Review Board of the Graduate School of Medicine, Osaka University (IBR No. 17,214).

\section{Next-generation sequencing (NGS)}

Genomic DNA was extracted from FFPE tissue using the QIAamp DNA FFPE Tissue Kit (Qiagen, Valencia, CA, USA) according to the manufacturer's instructions. Two pathologists (Y.H. and K.H.) selected FFPE blocks with greater than $50 \%$ abnormal tissue content in all cases. The gene panel was designed by SureDesign (https://earray.chem.agilent.com/suredesign) to cover a whole exon of 14 genes associated with the mTOR pathway signaling or responsible for other vascular anomalies (PIK3CA, TEK, GNA11, GNAQ, AKT1, PTEN, mTOR, CCM, BRAF, MAP3K3, KRAS, NRAS, HRAS, RASA1). On average $70 \mathrm{ng}$ of the extracted DNA was fragmented by SureSelect Fragmentation Enzyme (Agilent Technologies, Inc. Santa Clara, CA, USA ) to 150-200 bp. Sequence libraries were prepared with a custom SureSelect Low Input Target Enrichment System (Agilent Technologies, Inc. Santa Clara, CA, USA) according to the manufacturer's instructions and sequenced with the Illumina MiSeq (Illumina, San Diego, CA, USA). SureCall ver4.0 (https://www.agilent.com/en/download-softwaresurecall) was used for variant calling. DNA in introns or non-cording DNA were excluded. To confirm PIK3CA gene mutations, polymerase chain reaction (PCR) assays and direct sequencing were performed using the following primers: PIK3CA-Exon9 Forward, CAGCTCAAAG CAATTTCTAC; PIK3CA-Exon9 Reverse, CACTTACC TGTGACTCCAT; PIK3CA-Exon20 Forward, AACTGA GCAAGAGGCTTTGG; PIK3CA-Exon20 Reverse, TGTGTGGAAGATCCAATCCA. A mixture of 5\% PIK3CA-mutant DNA against a background of $95 \%$ wild-type (wt) DNA was used as a positive control.

\section{Histological and immunohistochemical staining}

Resected tissue samples were fixed with $10 \%$ formalin, routinely embedded in paraffin, cut into $4 \mu \mathrm{m}$ thick serial sections, and used for H\&E and immunohistochemical staining. Immunohistochemical staining was performed using a Roche Ventana BenchMark GX autostainer (Ventana Medical Systems, Tucson, AZ, USA) according to the manufacturer's instructions. Primary 
antibodies against p-AKT (\#4060, 1:100; Cell Signaling Technology, Danvers, MA, USA), p-mTOR (clone 49F9, 1:100; Cell Signaling Technology), p-S6K1 (\#9204, 1:100; Cell Signaling Technology), p-4EBP1 (clone 236B4, 1: 500; Cell Signaling Technology), S100 (polyclonal, Ventana Medical Systems), CD31 (clone JC70A, 1:200, Dako), CD34 (clone QBEnd10, 1:200, Dako), D2-40 (760-4395, Ventana Medical System), and PROX1 (ab199359, 1:500; Abcam, Cambridge, UK) were used. Samples were considered positive when at least $10 \%$ of the endothelial cells of abnormal vessels exhibited a signal for the targeted protein.

\section{Results}

\section{Clinical data and molecular genetic findings}

The four patients included four men, and they ranged in age from 3 to 15 years (median, 12.5 years). Two patients presented at birth (cases 1 and 3). The presenting symptoms were pain (4/4 cases), swelling (3/4 cases), and functional restriction (2/4 cases). The preoperative clinical diagnosis was vascular anomalies, including infantile hemangioma, vascular malformation, and FAVA. The lesions were located within and in the vicinity of the thigh muscles, and knee (Fig. 1 A-D). Heterogenous PIK3CA hotspot mutation (p.H1047R) was identified in two cases (cases 1 and 2) (Fig. 1E), while none of the PIK3CA mutations were detected in other two cases (cases 3 and 4) (Fig. $1 \mathrm{~F}$ ). We also found mutations in TEK in 2 cases, GNA11 in 1 case, AKT1 in 1 case, PTEN in 2 cases and HRAS in 1 case. The clinical characteristics and the results of the genetic analysis are summarized in Table 1.

\section{Histological and immunohistochemical findings}

The PIK3CA-mutation cases (cases 1 and 2) (Fig. 2 A-D) and PIK3CA-wt cases (cases 3 and 4) (Fig. 2E-H) possessed similar histology. All four cases exhibited abnormal vessels surrounded by dense fibrous tissue and adipose tissue with atrophic skeletal muscle (Fig. 2 A, E). The abnormal vessels were composed of $\mathrm{VM}$ and $\mathrm{LM}$ (Fig. 2B, F). The majority of the LM components possessed vascular clusters consisting of thin-walled backto-back blood-filled sacs (Fig. 2 C, G). The lymphatic phenotype was supported by endothelial D2-40 and/or Prox1 immunopositivity in consistent with our previous study [3]. In one PIK3CA-mutant case (case 2) and one PIK3CA-wt case (case 3), some nerves contained enlarged vessels (Fig. 2D, H). The endothelial cells of these vessels within nerves were positive for CD31 (marker for endothelial cells) and CD34 (marker for blood vessels), negative to weakly positive for PROX1 (marker for lymphatic vessels), and negative for D2-40 (marker for lymphatic vessels) (Fig. 2I). That is, the vessels within nerves had the vein-like characteristics. The other findings included the observation of organized thrombi within abnormal veins in two cases (cases 2 and 4) and lymphocytic aggregates surrounding abnormal vessels in three cases (cases 2-4). The histological findings of all cases are summarized in Table 2.

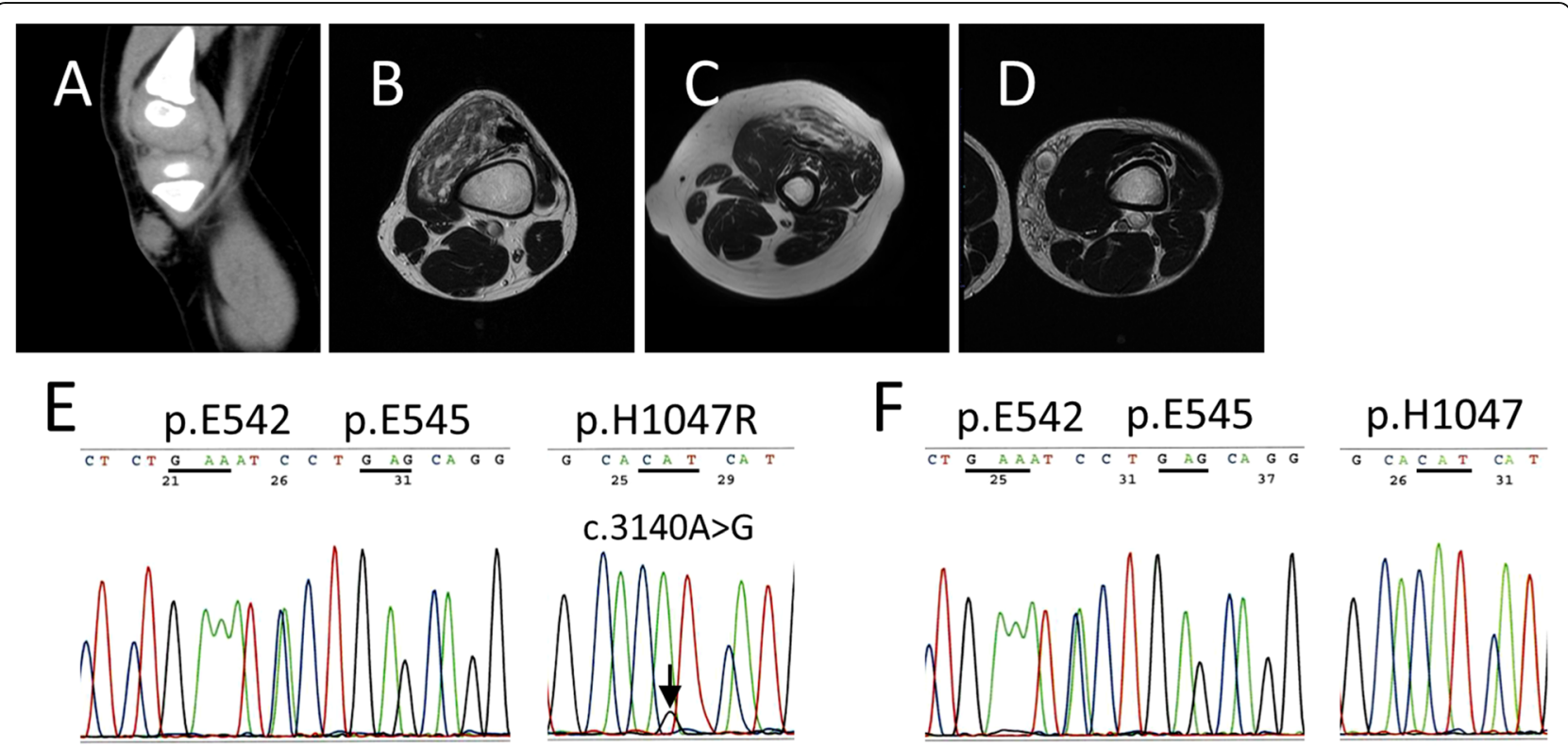

Fig. 1 A-D Computed Tomography (CT) or magnetic resonance image (MRIs) analysis. Sagittal CT image of case 1 (A), and axial T2-weighted MRI of case 2 (B), case $3(\mathbf{C})$ and case 4 (D). E, F DNA sanger sequencing of hotspot mutations in PIK3CA. Sequencing of PIK3CA for each FAVA cases showing chromatograms for c.3140 A>G (p.H1047R) (E) or wild-type (F) 
Table 1 Clinical and molecular genetic summary of the patients

\begin{tabular}{|c|c|c|c|c|c|c|c|}
\hline Case & Age/Sex & Location & Duration & Clinical diagnosis & Symptom & PIK3CA mutation & Other mutations \\
\hline 1 & $3 / M$ & Knee & 3 years & Infantile hemangioma & Pain, increased swelling & p.H1047R & PTEN (c.63dupG) \\
\hline 2 & $15 / M$ & Thigh muscle & $\begin{array}{l}3 \text { months (After } \\
\text { Sclerotherapy) }\end{array}$ & Vascular malformation & $\begin{array}{l}\text { Pain, functional restriction, } \\
\text { increased swelling, }\end{array}$ & p.H1047R & $\begin{array}{l}\text { TEK (p.W189*), GNA11 } \\
\text { (p.E191K), AKT1 } \\
\text { (p.R15Q, p.Y229=), PTEN } \\
\text { (c.63dupG), HRAS (p.R102W) }\end{array}$ \\
\hline 3 & $13 / M$ & Thigh muscle & 13 years & Venous malformation & Pain, increased swelling & No mutation & No mutation \\
\hline 4 & $12 / \mathrm{M}$ & Thigh muscle & 6 months & $\begin{array}{l}\text { Venous malformation } \\
\text { or FAVA }\end{array}$ & Pain, functional restriction & No mutation & TEK (p.R842H) \\
\hline
\end{tabular}

PIK3CA; phosphatidylinositol-4,5-Bisphosphate 3-Kinase Catalytic Subunit Alpha, TEK; TEK receptor tyrosine kinase, AKT1; AKT serine/threonine kinase 1, PTEN; phosphatase and tensin homolog, HRAS; HRas proto-oncogene, GTPase

We next examined the mTOR pathway activation status in abnormal vessels using immunohistochemical staining. The expression of p-AKT and p-mTOR, both of which are upstream of the mTOR pathway, was detected in abnormal vessels of the PIK3CA-mutation cases (cases 1 and 2) (Fig. 3 A, B) but not in those of the PIK3CA-wt cases (cases 3 and 4) (Fig. 3E, F). The expression of p-4EBP1 and p-S6K1, both of which are downstream effectors of the mTOR pathway, was detected in abnormal vessels of both PIK3CA-mutaion and PIK3CA-wt cases (Fig. 3 C, D, G, H). The one PIK3CAwt case (case 4) dose not express p-4EBP1 expression. In normal tissues, including the surrounding skeletal muscle and normal vessels, p-S6K1 exhibited sporadic expression, while $\mathrm{p}$-AKT, $\mathrm{p}$-mTOR, and $\mathrm{p}$-4EBP1 were not expressed at detectable levels. The immunohistochemical results are summarized in Table 3.

\section{Discussion}

FAVA is a new entity of vascular anomaly and is exceedingly rare. Alomari et al. (2014) [1] provided a proposed definition of the clinical and histological characteristics of FAVA. Subsequently, PIK3CA mutations were reported in a subset of FAVAs (5/8 cases) [4]. However, the correlations between specific mutations and clinicopathological features remain unclear. The current study is the first reported series of clinical, histological, immunohistochemical, and genetic analyses examining FAVA cases.

According to a clinical series of FAVA, FAVA arises in young patients (median age, 12-17 years) $[1,2]$. Common symptoms include pain (100\%), functional restriction $(81-100 \%)$, and swelling $(36.5 \%)[1,2]$. The most common location of the lesion is the lower extremities (94.7\%) [2]. Our current study determined that the median age of PIK3CA-mutation patients was 9 years and that of PIK3CA-wt patients was 12.5 years. Both PIK3CA-mutation and PIK3CA-wt patients presented with pain, swelling, and functional restriction. All four lesions were located in the lower extremities. Based on the above findings, our cases exhibited typical clinical features regardless of PIK3CA mutational status (Table 1) $[1,2]$. Similarly, both PIK3CA-mutation and PIK3CA-wt cases exhibited the typical histological features of FAVA (Table 2). Histologically, VM, LM, fibrous tissue, and adipose tissue were observed in all cases. Nerves containing enlarged vessels, a condition that is unusual in other vascular anomalies, were also present regardless of PIK3CA mutational status (Fig. 2D, H). Thus, our observations indicated that there was no significant association between the presence of PIK3CA mutations and the clinicopathological features of FAVA.

Immunohistochemical analysis showed that p-AKT and p-mTOR that act upstream of the mTOR pathway were detected in abnormal vessels of PIK3CA-mutation cases, but not in those of PIK3CA-wt cases (Table 3). On the other hand, $\mathrm{p}-4 \mathrm{EBP} 1$ and $\mathrm{p}-\mathrm{S} 6 \mathrm{~K} 1$, downstream of the mTOR pathway, were detected in abnormal vessels of both PIK3CA-mutation and PIK3CA-wt cases (Table 3). One interpretation of this discrepancy was that 4EBP1 and S6K1 were activated in mTORindependent manner. In fact, phosphorylation of 4EBP1 and S6K1 is subject to mTOR-independent several kinases and feedback loops $[14,15]$. Somatic mutations in PIK3CA occur frequently in cancers other than LMs and other PIK3CA-related overgrowth spectrums [4-6, 8]. In cancers, a small number of studies have demonstrated a positive correlation between PIK3CA mutational status and upstream activation of the mTOR pathway [16-18]. p-AKT and p-mTOR were immunohistochemically expressed more frequently in PIK3CA-mutation cases than in PIK3CA-wt cases, while the immunohistochemical expression of $\mathrm{p}-4 \mathrm{EBP} 1$ and $\mathrm{p}-\mathrm{S} 6 \mathrm{~K} 1$ was not correlated with the presence of PIK3CA mutation [16-18]. These results were consistent with the relationship between the mutation and immunohistochemical expression in our FAVA cases.

Both 4EBP1 and S6K1 are involved in the development of abnormal vessels in VMs and LMs by promoting protein synthesis and cell growth $[5-9,11,12]$. The activation of 4EBP1 and S6K1 may play a key role in the pathogenesis of abnormal vessels in FAVA lesions; 


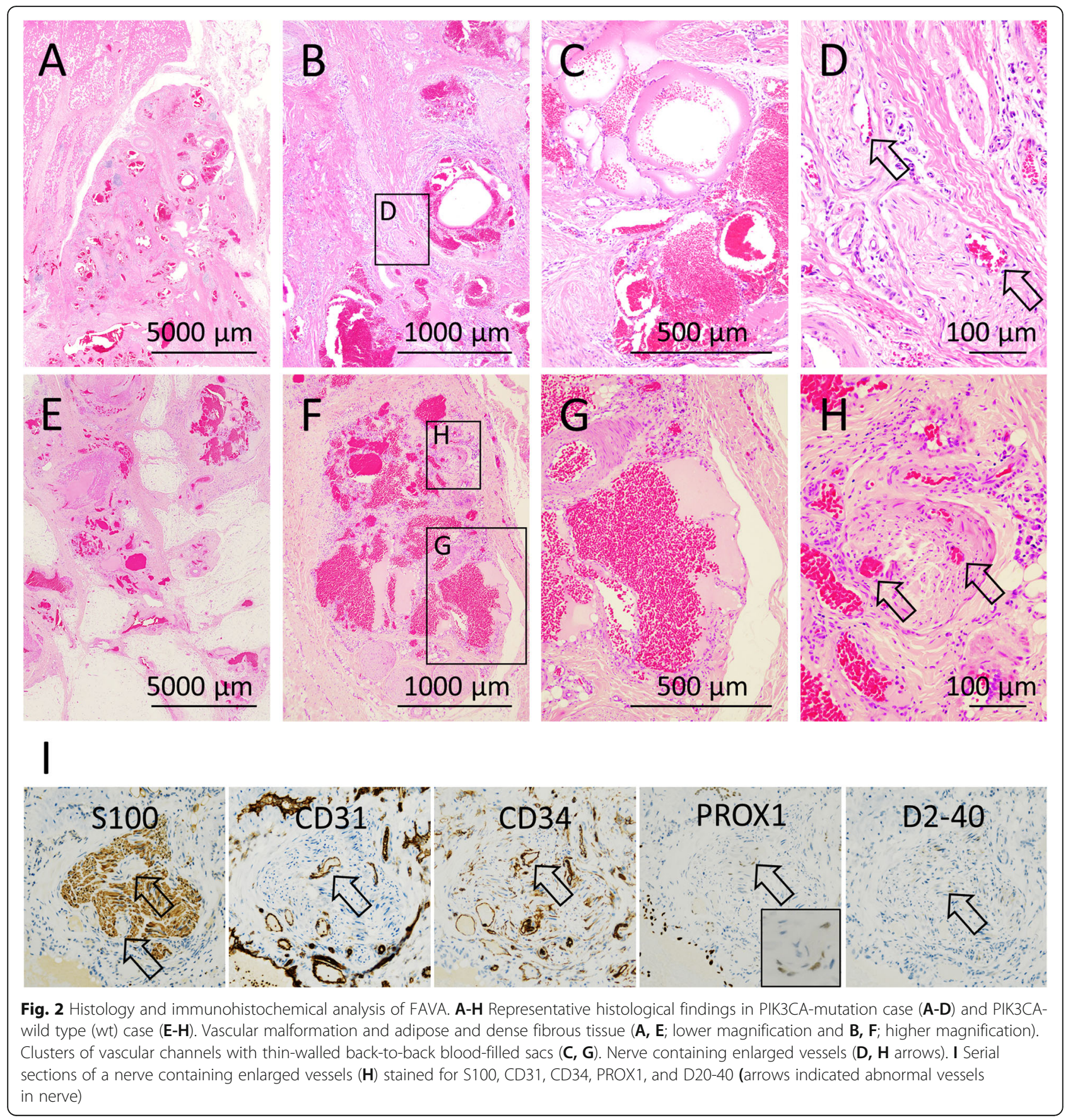

Table 2 Summary of histological findings

\begin{tabular}{llllll}
\hline Case & VM & LM & Nerve containing enlarged abnormal vessels & Organized thrombi & Lymphocytic aggregates \\
\hline 1 & + & + & - & - & - \\
2 & + & + & + & + & + \\
3 & + & + & + & - & + \\
4 & + & + & - & + & + \\
\hline
\end{tabular}




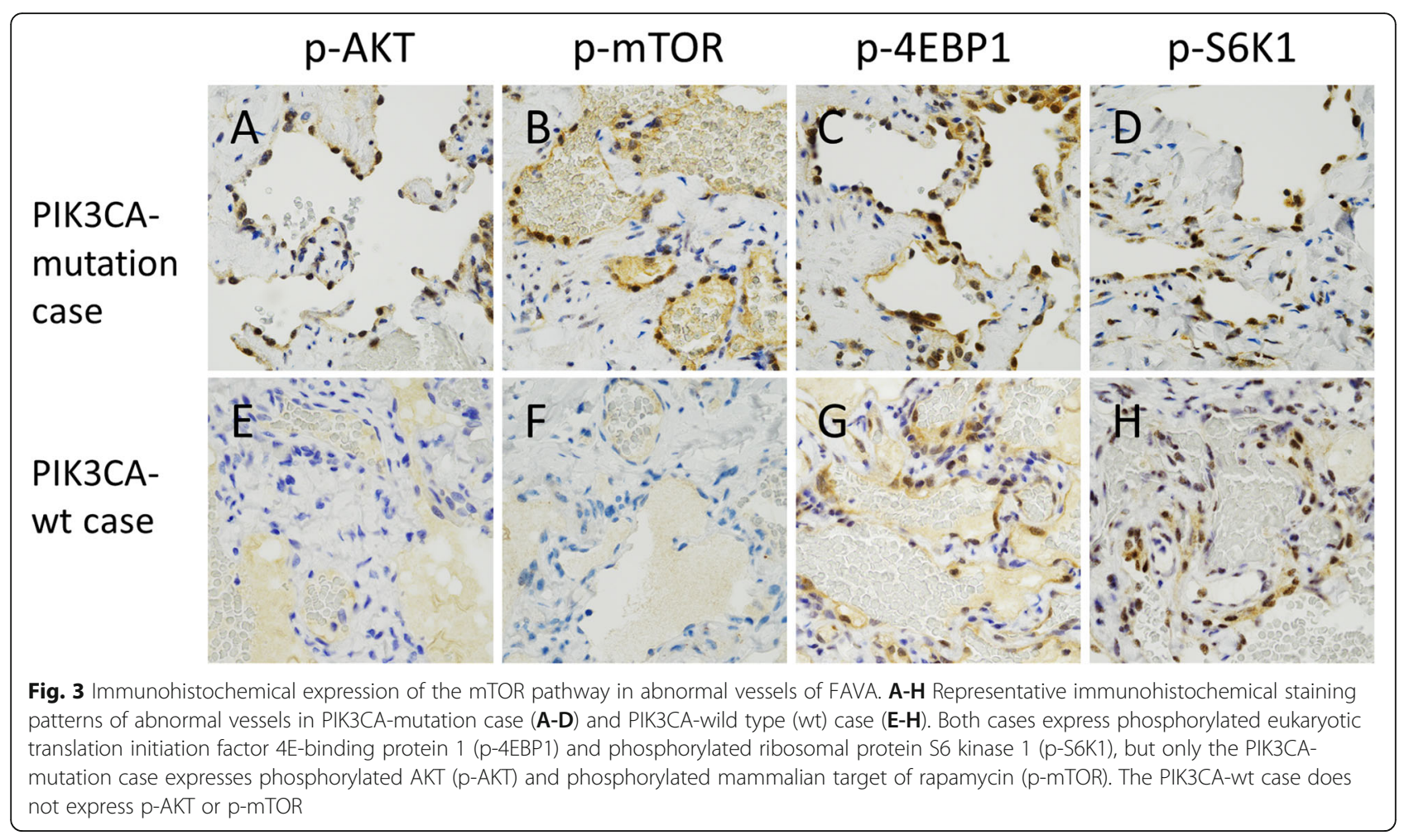

however it was unclear what signaling pathways were involved in their activation. Since the identification of PIK3CA mutations in FAVA by Luks et al. [4], further mutational analyses of FAVA have not been performed. Our targeting NGS failed to identify common gene mutations associated with mTOR pathway among PIK3CAwt cases, although TEK mutation (p.R842H within exon 13) was detected in one PIK3CA-wt case. Somatic gainof-function mutations in TEK gene that encodes the endothelial tyrosine-protein kinase receptor TIE-2 occurs approximately half of sporadic VMs and in a subset of LMs $[8,19,20]$. TEK hotspot mutations are detected exclusively in exon 17 and are present within the first tyrosine kinase and kinase insert domains of the receptor $[8,19,20]$. TEK hotspot mutations result in a constantly active PI3K/AKT signaling pathway involving angiogenesis $[8,19,20]$. On the other hand, AKT phosphorylates

Table 3 Immunohistochemical expression of mTOR pathway in abnormal vessels

\begin{tabular}{lllll}
\hline Case & p-AKT & p-mTOR & p-4EBP1 & p-S6K1 \\
\hline 1 & + & + & + & + \\
2 & + & + & + & + \\
3 & - & - & + & + \\
4 & - & - & - & + \\
\hline
\end{tabular}

Staining intensity (-; no expression / +; positive) many downstream molecules involved in the regulation of cellular functions. Therefore, little is known about the association with TEK mutations and activation of mTOR downstream effectors in VMs. The identified TEK p.R842H (c.2525G>A) mutation in current study is reported in the COSMIC (Catalogue of Somatic Mutations in Cancer) database, however the function of this mutation is not investigated. Considering that activated AKT was not detected in PIK3CA-wt cases, the TEK p.R842H mutation may not activate AKT in FAVA. Approximately $25 \%$ of VMs lacked both TEK and PIK3CA mutations $[8,10]$, and the responsible genetic aberrations remain unclear. Thus, FAVA lacking PIK3CA mutations may be caused by undiscovered mutations that activate 4EBP1 and S6K1.

\section{Conclusions}

In this study, we reported the results of clinical, histological, immunohistochemical, and genetic analyses examining a small series of isolated FAVA. There was no significant association between the presence of PIK3CA mutations and the clinical and histological features of FAVA, suggesting that the PIK3CA gene may be not necessarily involved in the onset of FAVA. FAVA lacking PIK3CA mutations may be caused by other mutations that activate 4EBP1 and S6K1. 


\section{Abbreviations}

4EBP1: Eukaryotic translation initiation factor 4E-binding protein; AKT1: AKT serine/threonine kinase 1; BRAF: B-Raf proto-oncogene, serine/threonine kinase; FAVA: Fibro-adipose vascular anomaly; FFPE: Formalin-fixed paraffinembedded; GNA11: G protein subunit alpha 11; GNAQ: G protein subunit alpha q; HRAS: HRas proto-oncogene, GTPase; KRAS: KRAS proto-oncogene, GTPase; KTS: Klippel-Trenaunay syndrome; LM: Lymphatic malformation; MAP3K3: Mitogen-activated protein kinase kinase kinase 3;

mTOR: Mammalian target of rapamycin; NRAS: NRAS proto-oncogene, GTPase; PCR: Polymerase chain reaction; PI3K: Phosphoinositide 3-kinase; PIK3CA: Phosphatidylinositol-4, 5-bisphosphate 3-kinase catalytic subunit alpha; PTEN: Phosphatase and tensin homolog; RASA1: RAS p21 protein activator 1; S6K1: Ribosomal protein S6 kinase 1; TEK: TEK receptor tyrosine kinase; VM: Venous malformation; WT: Wild-type

\section{Acknowledgements}

Not applicable.

\section{Authors' contributions}

All authors contributed to this work. Yumiko $\mathrm{H}$. and Katsutoshi $\mathrm{H}$. participated in the concept of this study and wrote the manuscript. These two authors contributed equally to this work. Yumiko H., Katsutoshi H., Michio O., Kenji H., Daisuke M., Shinichro T., Takahiro M., Masaharu K. and Satoru T. performed the experiments and assembled data. Yumiko $\mathrm{H}_{4}$ Katsutoshi $\mathrm{H}$. and Eiichi $\mathrm{M}$. were critically revised the manuscript for intellectual content. Hiroki H., Yusuke O. and Kaishu T. reviewed the clinical and radiological data. All authors reviewed and approved the manuscript for submission.

\section{Funding}

This work was supported by JSPS KAKENHI (21K15384) and Practical Research Project for Rare / Intractable Diseases (21ek0109515h0001) from Japan's Agency for Medical Research and Development, AMED.

\section{Availability of data and materials}

The surgical materials and the datasets analyzed during the current study are available from the corresponding author on reasonable request.

\section{Declarations}

\section{Ethics approval and consent to participate}

This study was approved by the Ethical Review Board of the Graduate School of Medicine, Osaka University (No. 17214), and was performed in accordance with the Committee guidelines and regulations.

\section{Consent for publication}

Written informed consent for publication of their clinical details and clinical images was obtained from the patients. A copy of the consent form is available for review by the Editor of this journal.

\section{Competing interests}

The authors declare no conflicts of interest.

\section{Author details}

'Department of Pathology, Osaka University Graduate School of Medicine, 2-2 Yamadaoka, 565-0871 Suita, Osaka, Japan. ²Department of Central Laboratory and Surgical Pathology, National Hospital Organization, Osaka National Hospital, 2-1-14 Hoenzaka, Chuo-ku, Osaka-shi 540-0006, Osaka, Japan. ${ }^{3}$ Department of Oral Pathology, Osaka University Graduate School of Dentistry, 1-8 Yamadaoka, 565-0871 Suita, Osaka, Japan. ${ }^{4}$ Department of Pediatrics, Graduate School of Medicine, Gifu University, 1-1 Yanagido, 501-1194 Gifu, Japan. ${ }^{5}$ Department of Molecular and Cellular Biochemistry, Osaka University Graduate School of Dentistry, 1-8 Yamadaoka, 565-0871 Suita, Osaka, Japan. ${ }^{6}$ Genome Information Research Center, Research Institute for Microbial Diseases, Osaka University, 3-1 Yamadaoka, 565-0871 Suita, Osaka, Japan. ${ }^{7}$ Department of Radiology, Osaka University Graduate School of Medicine, 2-2 Yamadaoka, 565-0871 Suita, Osaka, Japan.
Received: 14 October 2021 Accepted: 15 January 2022

Published online: 30 January 2022

\section{References}

1. Alomari Al, Spencer SA, Arnold RW, et al. Fibro-adipose vascular anomaly: clinical-radiologic-pathologic features of a newly delineated disorder of the extremity. J Pediatr Orthop. 2014;34:109-17.

2. Amarneh M, Shaikh R. Clinical and imaging features in fibro-adipose vascular anomaly (FAVA). Pediatr Radiol. 2020;50:380-87.

3. Hori Y, Hirose K, Aramaki-Hattori N, et al. Fibro-adipose vascular anomaly (FAVA): three case reports with an emphasis on the mammalian target of rapamycin (mTOR) pathway. Diagn Pathol. 2020;15:98.

4. Luks VL, Kamitaki N, Vivero MP, et al. Lymphatic and other vascular malformative/overgrowth disorders are caused by somatic mutations in PIK3CA. J Pediatr. 2015:166:1048-54.e1-5.

5. Madsen RR, Vanhaesebroeck B, Semple RK. Cancer-Associated PIK3CA Mutations in Overgrowth Disorders. Trends Mol Med. 2018:24:856-70.

6. Keppler-Noreuil KM, Rios JJ, Parker VE, et al. PIK3CA-related overgrowth spectrum (PROS): diagnostic and testing eligibility criteria, differential diagnosis, and evaluation. Am J Med Genet A. 2015;167A:287-95.

7. Blesinger $\mathrm{H}$, Kaulfuß S, Aung T, et al. PIK3CA mutations are specifically localized to lymphatic endothelial cells of lymphatic malformations. PLoS One. 2018;13:e0200343.

8. Castillo SD, Baselga E, Graupera M. PIK3CA mutations in vascular malformations. Curr Opin Hematol. 2019;26:170-78.

9. Castel P, Carmona FJ, Grego-Bessa J, et al. Somatic PIK3CA mutations as a driver of sporadic venous malformations. Sci Transl Med. 2016;8:332ra42.

10. Limaye N, Kangas J, Mendola A, et al. Somatic Activating PIK3CA Mutations Cause Venous Malformation. Am J Hum Genet. 2015;97:914-21.

11. Whalen SG, Gingras AC, Amankwa $L$, et al. Phosphorylation of elF-4E on serine 209 by protein kinase $C$ is inhibited by the translational repressors, 4E-binding proteins. J Biol Chem. 1996;271:11831-7.

12. Dennis PB, Pullen N, Kozma SC, et al. The principal rapamycin-sensitive p70(s6k) phosphorylation sites, T-229 and T-389, are differentially regulated by rapamycin-insensitive kinase kinases. Mol Cell Biol. 1996;16:6242-51.

13. Zenner K, Cheng CV, Jensen DM, et al. Genotype correlates with clinical severity in PIK3CA-associated lymphatic malformations. JCI Insight. 2019;4: e129884.

14. Qin X, Jiang B, Zhang Y. 4E-BP1, a multifactor regulated multifunctional protein. Cell Cycle. 2016;15:781-86.

15. Arif $A$, Jia J, Willard B, Li X, Fox PL. Phosphorylation of S6K1 directs a kinase phospho-code that determines substrate selection. Mol Cell. 2019;73:446-57.

16. Maruyama N, Miyoshi Y, Taguchi T, et al. Clinicopathologic analysis of breast cancers with PIK3CA mutations in Japanese women. Clin Cancer Res. 2007; 13(2 Pt 1):408-14.

17. Azim HA, Kassem L, Treilleux I, et al. Analysis of PI3K/mTOR Pathway Biomarkers and Their Prognostic Value in Women with Hormone ReceptorPositive, HER2-Negative Early Breast Cancer. Transl Oncol. 2016:9:114-23.

18. Wang J, Zhu X, Xu X, et al. PIK3CA mutations and downstream effector $p$ mTOR expression: implication for prognostic factors and therapeutic targets in triple negative breast cancer. Int J Clin Exp Pathol. 2017;10:7682-91.

19. Limaye $\mathrm{N}$, Wouters $\mathrm{V}$, Uebelhoer $\mathrm{M}$, et al. Somatic mutations in angiopoietin receptor gene TEK cause solitary and multiple sporadic venous malformations. Nat Genet. 2009:41:118-24.

20. Ye C, Pan L, Huang Y, Ye R, Han A, Li S, Li X, Wang S. Somatic mutations in exon 17 of the TEK gene in vascular tumors and vascular malformations. J Vasc Surg. 2011;54:1760-8.

\section{Publisher's Note}

Springer Nature remains neutral with regard to jurisdictional claims in published maps and institutional affiliations. 